\title{
The Effects of Social and Spatial Presence on Engagement in a 3D Virtual Reality Environment: An Empirical Investigation
}

\author{
Supavich (Fone) Pengnate \\ College of Business \\ North Dakota State University \\ fone.pengnate@ndsu.edu
}

\author{
Frederick J. Riggins \\ College of Business \\ North Dakota State University \\ fred.riggins@ndsu.edu
}

\author{
Limin Zhang \\ College of Business \\ North Dakota State University \\ limin.zhang@ndsu.edu
}

\begin{abstract}
Virtual reality (VR) provides opportunities for businesses to innovatively engage customers. Based on presence theory, a research model was developed to test the influence of two major components of presence, social presence and spatial presence, on users' perceptions of hedonic value, utilitarian value, and engagement. An experiment was conducted on two conditions of a VR application (low vs. high social presence) to test the hypotheses in the research model. The results reveal that social presence and spatial presence can improve hedonic value of VR. However, inconsistent with previous studies, our findings reveal a negative relationship between spatial presence and engagement. Theoretical and practical implications, as well as future research directions are subsequently discussed.
\end{abstract}

\section{Introduction}

The explosive evaluation of computer-mediated technology in recent years has fostered the proliferation of advanced technologies that enable businesses to provide better services to customers. In this study, we explore an application of a fully immersive virtual reality (VR) experience in the business context. VR is considered as an immersive technology that is expected to become mainstream for providing services in several industries within the next few years [1]. In general, the major goal of VR is to improve user experiences because this technology provides high levels of immersion that enables users to experience the feeling of being in another location while using VR services [2]. In recent years several companies have invested millions of dollars in VR applications and services for advertising, meetings, trainings, and simulations. Business investment in VR is expected to increase from $\$ 9.1$ billion in 2017 to $\$ 17.8$ billion in 2018 [3].
A wide range of academic disciplines are interested in VR and its applications. In the past decades, business organizations have evolved how they provide products and services to customers particularly regarding the use of information technology. Because of advancements in technology, online companies have strived to deliver more interactive and engaging services to improve customer experiences. Currently, technologies such as VR have enabled business organizations to provide their customers with higher levels of immersion and interactivity than ever before. Therefore, VR has been of interest to both academics and practitioners. However, the current research focus has mostly been on developing VR applications or exploring opportunities for applying VR into specific areas. For example, Padmanaban et al. [4] suggest a method to improve imagery quality, Greenwald et al. [5] examine the use of VR in educational settings, and Marquess et al. [6] investigate how VR can help reduce patients' anxiety in receiving radiation therapy.

Despite high expectations and popularity, regarding the theoretical frameworks related to VR prior research has mostly focused on 2D web-based VR technologies (e.g., virtual worlds) that provide much less immersive experience and interaction with the virtual environment than the recently developed fully immersive VR. Therefore, this technology is still in its infancy and the mechanisms that shape users' behavioral responses by increasing the levels of immersion remain largely unexplored.

Consequently, this study highlights the affective and cognitive aspects of VR experience to address the potential factors that lead to user engagement with VR applications from the user's perspective. We focus on how users perceive VR based on users' evaluations rather than from the properties being pre-embedded by the developers [7].

The contributions of this paper are two-fold. First we aim to examine how social presence and spatial presence, which are key features in the virtual world research [8], influence user responses in fully 
immersive VR settings. We develop a research model to theorize the relationships between VR features related to the presence concepts and user engagement. By investigating such relationships, we aim to identify new research opportunities arising from the applications of VR, especially regarding the underlying mechanism between $\mathrm{VR}$ and user engagement. Second, while VR provides many features for businesses to attract their customers, we aim to explore the features that have potential to better engage customers, which subsequently help practitioners make informed decision about which features to design and implement in order to improve their customer experiences. As such, our paper is relevant not only for researchers in information systems but practitioners in related business functions such as marketing, operations, and human resources who are interested in applying VR to their area.

This paper proceeds as follows. Section 2 surveys the relevant literature of VR and user engagement, and outlines hypothesis development and our research model derived from existing theories. Section 3 presents the proposed research method and detailed experiment design, followed by data analysis and results in Section 4. Finally, Section 5 provides a summary of results, contributions, and future directions.

\section{Literature review and hypothesis development}

\subsection{Virtual reality}

Virtual reality (VR) refers to a computer-mediated system that interacts with its users by providing an immersive environment and experience [9]. Typically, VR provides users with a higher degree of immersion in a computer-mediated environment than traditional computer systems. This feeling of immersion in an environment has been referred to as the perceptions of presence [10].

The applications of VR for home use have just started to emerge. Although past studies have investigated VR applications in the business context, they often restrict VR as 2D web-based applications that run on desktop computers or mobile devices (referred to as 2D web-based VR) that provide users with an internet-based simulated virtual environment. In such technologies, users are generally represented by avatars in the VR environment and usually do not see a visual representation of themselves [11]. Although studies in 2D web-based VR can shed some light on the understanding of how the virtual environment affects customers' perceptions, such settings provide much less immersive experience in terms of consciousness, senses and interactions with the environments than the fully immersive $3 \mathrm{D} V R$ systems (referred to as VR) in which the environment is fully generated by computers. Thus, VR provides wider and broader information that can better manipulate and stimulate the human senses than $2 \mathrm{D}$ web-based VR.

\subsection{Presence theory}

Proposed by Loomis [12], presence is described as an attribution of sensation to distal stimulus that evokes a basic state of consciousness in a certain environment. According to Ijsselsteijn et al. [13], presence can be classified into two categories - spatial presence and social presence. Spatial presence can be commonly referred to as "a sense of being there." This definition is identical to the concept of telepresence previously studied in the literature [14]. In the context of VR, spatial presence can occur when a user's perceptions of the environment created by VR are different from the actual location and environment in the physical world.

On the other hand, social presence can be conceptualized as a capability of a medium which causes users to perceive the presence of others [15]. In this study, social presence refers to the degree to which a user establishes a sense of human warmth and sociability while interacting with a medium [16]. Previous studies in the $2 \mathrm{D}$ web-based VR have reported that incorporating human-like objects in the virtual environments can improve users' perceptions of social presence [17]. Consequently, in our study, we manipulate the levels of social presence in the VR environment by using an animated robot assistant (as a VR component) to create experimental conditions for a VR environment. This should help us better understand how design and feature components in VR impact users' perceptions of social presence. Further details regarding the robot assistant are provided in the methodology section. Consequently, we propose the following hypothesis:

H1: Users' perceptions of social presence will be higher when a robot assistant is present in the VR environment.

\subsection{Consequences of presence}

In this study, we focus on the user's perspective and seek to explain how VR features motivate users to engage in the virtual environments. Therefore, we decided to adopt the human motivation framework 
proposed by Deci and Ryan [18] that explains the mechanisms underlying users' motivations and choices of actions.

Deci and Ryan [18] proposed that human motivations can be driven by two major factorsintrinsic motivation and extrinsic motivation. While intrinsic motivation refers to an individual's desire to obtain a feeling of pleasure, extrinsic motivation refers to the performance of an activity in order to attain a desired outcome. The values of intrinsic and extrinsic motivations have been reported as key determinants of users' behaviors [19]. Overall, these values are derived from the attributes of a system and can be classified as hedonic value (corresponding to intrinsic motivation) or utilitarian value (corresponding to extrinsic motivation). The notion of human motivation provides a reasonable linkage between system attributes, values, and behavior. Therefore, in this study, we propose that attributes of VR (social presence and spatial presence) can influence values of VR (hedonic value and utilitarian value), which further motivate users' choice of behavior (engagement).

Regarding the social dimension of presence, previous studies have suggested that interactions among online customers can evoke positive emotions (e.g., enjoyment) which subsequently improve online shopping experience [20]. This notion is supported by a strong research stream in marketing that has found that social factors in a stimulus can influence customers' positive emotions and responses, especially in online stores [21]. Therefore, we propose that if users experience high social presence in VR applications, they would feel that VR is more pleasurable to use; hence, the level of hedonic value increases.

H2: Users' perception of social presence is positively related to their perception of hedonic value.

In addition, by accelerating users' immersion and interaction, VR can better provide a sense of sociability which often results in engagement. For example, in the 2D web-based VR area, Fortin and Dholakia [22] reported a positive relationship between social presence and engagement. Therefore, we propose the following hypothesis:

H3: Users' perception of social presence is positively related to their engagement with VR.

As demonstrated by previous studies, social presence can influence utilitarian value because it presents other users or sociable objects at a higher level of immersion [23]. Thus, this can help reduced ambiguity and uncertainty in performing tasks, which consequently increases the hedonic value of VR [24, 25]. Therefore, we propose the following:

H4: Users' perception of social presence is positively related to their perception of utilitarian value.

Prior research in online commerce suggest that spatial presence can be determined by information about physical stores, products on webpages, and consumers' recall of those physical stores and products [26]. Spatial presence was reported to influence positive effects on users' responses through positive emotions [27, 28].

In this study, spatial presence is induced by the components in VR which include the $3 \mathrm{D}$ objects and the environment that users experience. According to Shin [29], spatial presence can increase the level of the immersion of users involvement [30] through positive emotions (e.g., enjoyment), which subsequently lead to engagement [31]. Therefore, in line with these studies, we propose that spatial presence in the VR context also positively influences hedonic value and engagement.

H5: Users' perception of spatial presence is positively related to their perception of hedonic value.

H6: Users' perception of spatial presence is positively related to their engagement with VR.

Spatial presence has been consistently reported to positively influence usefulness of computer-mediated technologies. Held and Durlach [32] found that spatial presence can increase performance of the subjects in their experiment. Apparently, VR can provide users with several features that promote utilitarian value dimensions. For example, the richness of product information available in the VR environment can positively influence users' efficiency [33]. Therefore, spatial presence is expected to enhance utilitarian value of the VR application.

H7: Users' perception of spatial presence is positively related to their perception of utilitarian value.

Drawing from the research area of humancomputer interaction, engagement has emerged as a critical factor created by the interactions between a computer system and its users that influence the users' subsequent responses [34]. According to Csikszentmihalyi [30], both hedonic and utilitarian 
motivations are considered as determinants of cognitive engagement. Both motivations are characterized as intrinsic motivations for users to respond to new stimuli and a willingness to try new activities [35]. Therefore, we propose the following hypotheses:

H8: Users' perception of hedonic value is positively related to their engagement with VR.

H9: Users' perception of utilitarian value is positively related to their engagement with VR.

\section{Methodology}

\subsection{Experimental procedure, task and measures}

A between-subject research design experiment was conducted in VR to test the proposed hypotheses. Participants were randomly assigned to one of the two experimental conditions to complete the experimental task of evaluating a new hypothetical product in VR.

Specifically, the experimental task was designed to use VR to perform an evaluation of a new productan empty beverage can package (VR object). Participants were asked to perform a utilitarian-based task which included evaluating the product in terms of its innovation, quality, and visual appeal, and then completing a survey to provide their feedbacks about the product. Participants were guided to freely interact with the product such as picking up, holding, rotating, and placing the product on a table.

Regarding the manipulation of social presence, two experimental conditions were created to manipulate the interaction with a robot assistant (VR object). The robot assistant was presented in both experimental conditions. However, in Condition 1 (low social presence), participants were asked to perform the task without activating the robot. In Condition 2, participants were asked to interact with the robot to evoke the sense of social presence. The robot was programmed to provide participants with human-like social interactions such as greeting, waving hands, and expressing certain emotions (e.g., fear, surprised, and happiness). Note that the robot assistant was not directly involved in the main experiment task which was to evaluate the new product.

Prior to performing the experimental task in either low or high social presence conditions, participants had to complete a tutorial pertaining to the use of the VR system (Oculus Rift ${ }^{\mathrm{TM}}$ ), which included a head mount display (HMD) and hand controllers. This tutorial was implemented by walking through the instructions in a VR application provided by Oculus $^{\mathrm{TM}}$. Participants were then randomly assigned to one of the two experimental conditions to perform the task. Upon finishing the task, participants completed a questionnaire that measured the following constructs: social presence, spatial presence, hedonic value, utilitarian value, and engagement. All the items in the questionnaire were derived from existing studies. Social presence was measured by three items adapted from Hassanein and Milena [16]), spatial presence was measured using four items adapted from Ahn et al. [36], hedonic value was measured using three items adapted from Zhou et al [37], utilitarian value was measured using three items adapted from Zhou et al. [37], and engagement was measured by six items adapted from Wiebe et al. [38]. Table 1 presents the questionnaire items. The items were measured by seven-point Likert scales with a score of 1 to 7 (1 corresponding to "totally disagree" and 7 to "totally agree"). Each experimental session lasted approximately 30 minutes.

Table 1. Questionnaire items

Social Presence [16]

- There was a sense of human warmth in this VR app.

- There was a sense of sociability in this VR app.

Spatial Presence [36]

- While I was evaluating the product in VR, I felt like I was in the real environment.

- While I was evaluating the product in VR, I felt like I was surrounded by the real objects.

- While I was evaluating the product in VR, the environment in VR seemed like the real world.

Hedonic Value [37]

- Overall, the use of this VR app gave me pleasure.

- I enjoyed being immersed in the environment while using this VR app.

Utilitarian Value [39]

- I finished the tasks I initially intended to do in this VR app.

- I accomplished what I initially wanted to do in this VR app.

Engagement [38]

- While I was using VR to evaluate the product, I lost track of the world around me.

- While I was using VR to evaluate the product, I blocked out things around me.

- While I was using VR to evaluate the product, the time passed slowly. (reversed question) 


\subsection{Sampling and participant information}

A total of 38 usable responses was collected from a sample of undergraduate students (10 females and 28 males) who were enrolled in a midwestern university in the United States. Each experimental condition contained 19 responses. Approximately $60 \%$ of the participants were between 21-23 years old. Fifty percent of the participants had previously used VR at least once before the experiment was conducted and about $10 \%$ owned a VR headset.

\section{Results}

\subsection{Measurement model}

A confirmatory factor analysis (CFA) was performed to assess the validity of the measures. Consequently, construct validity and reliability of the measurement model were assessed using composite reliability (CR) and Cronbach's alpha. The results reveal that $C R$ values ranged from 0.766 to 0.928 and the Cronbach's alpha ranged from 0.626 to 0.883 . Both the CR and Cronbach's alpha values are above the acceptable level suggested by Hair et al. [40]. Thus, the constructs demonstrate good construct reliability and convergent validity.

Discriminant validity of the constructs was then evaluated. The results indicate that the square root of the variance shared between a construct and its measurement items ranged from 0.745 to 0.900 and these are greater than the correlations between the construct and other constructs in the model which ranged from 0.061 to 0.513 ; therefore, the measures satisfy the criteria for discriminant validity [40].

\subsection{Manipulation check}

A manipulation check was performed to ensure that the experimental conditions (low vs. high social presence) were manipulated effectively and the results suggest that the manipulations on the degrees of social presence were deemed successful.

\subsection{Social presence: Hypothesis testing}

An independent sample t-test was conducted to test hypothesis $\mathrm{H} 1$ for the difference in users' perception of social presence in the experimental conditions. The results suggest that users completing the experimental task in the condition with the interaction with the robot assistant (Condition 2) perceived that it has a higher degree of social presence than in the condition without the interaction with the robot assistant (Condition 1) (MeanCond $1=4.1 ;$ SDCond $1=1.45$ vs. MeanCond $2=6.32 ;$ SDCond $2=0.88 ; \mathrm{p}<0.001$ ); thus, H1 is statistically supported. The remaining hypotheses $(\mathrm{H} 2-\mathrm{H} 9)$ were tested in a structural model using the partial least square (PLS) technique.

\subsection{Structural equation model: Hypothesis testing}

A structural equal modeling was conducted to test Hypotheses $2-9$ proposed in the research model. As shown in Figure 1, approximately $18 \%$ of the variance was explained by the exogenous variables. In particular, relationships predicted in Hypotheses H5 and $\mathrm{H} 9$ are statistically significant at the 0.05 level. Spatial presence positively influences hedonic value and utilitarian value positively affect engagement. However, the results also reveal interesting findings that the relationship between spatial presence and engagement is significant at the level of 0.05 (H6: standardized coefficient $=-0.313$ ), but the direction is opposite compared to the results from previous studies that hypothesize a positive effect of spatial presence on engagement.

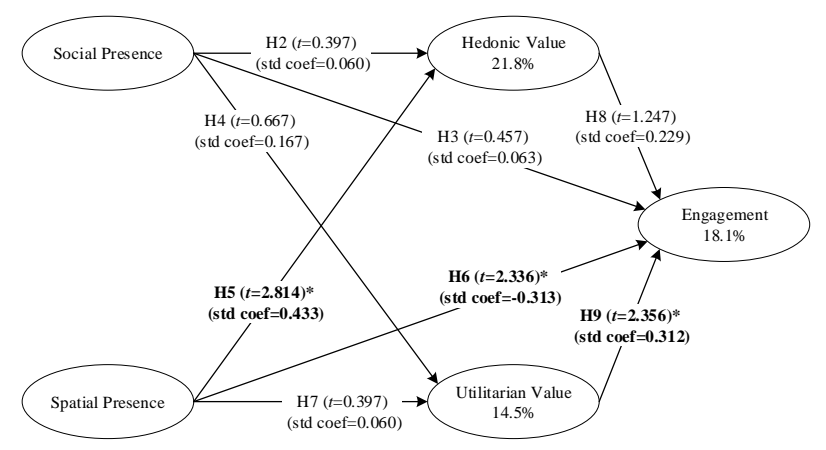

Figure 1. Research model, $t$-value, and standardized coefficients $(*$ p-value $<0.05)$

\section{Discussion and conclusion}

\subsection{Summary of the results}

This study examines the factors that influence users' perceptions toward the use of VR in product evaluation. Specifically, we investigate how social presence and spatial presence, the two major features based on presence theory in the VR context, influence users' perceptions and responses, which consists of hedonic value, utilitarian value, and engagement. Our results reveal several interesting findings. 
First, according to hypothesis H1, the perception of social presence emerged when the robot assistant was presented in the VR environment. Thus, consistent with findings from previous studies in the 2D web-based virtual world environment [41], this study found that the level of social presence in a highly immersive environment (e.g., VR) is also affected by the presence of computer-generated sociability objects, such as the robot assistant in our study.

Second, as shown in the research model, the results suggest that spatial presence is a more important predictor of hedonic value than social presence (H2 vs. H5). These findings are consistent with previous studies that investigated these relationships in the online commerce context [23]. Therefore, this study confirms the results that VR users enjoy virtual environments that are capable of providing senserelated ambient cues or a perceptual illusion of "being there."

Third, according to the relationship between spatial presence and engagement (H6), the results indicate that higher levels of realism in the virtual environment can reduce user engagement, which contradict those reported in prior studies [31, 42]. A possible explanation is that the relationship between spatial presence and engagement might not be linear, but rather an inverted U-shaped function as often reported when users' emotional responses were investigated [43].

Finally, considering the effects of hedonic and utilitarian values on engagement (H8 and H9), the findings reveal that utilitarian value is a more effective predictor of engagement. Therefore, these results are in line with previous studies in the area of technology adoption [44] suggesting that utilitarian value (e.g., usefulness) outperformed hedonic value in determining user responses.

\subsection{Theoretical contributions}

A major theoretical contribution of this research is to empirically test the effects of presence on VR users. While such effects were tested in other computermediated technology domains (e.g., 2D web-based VR and online commerce), this study further enhances existing knowledge by extending the applicability of presence theory to the fully immersive 3D VR context. In line with prior studies in other related areas, our results confirm such effects of presence in the VR settings. This effort not only further highlights the value of incorporating features related to presence in VR applications but also contributes to the literature by adapting similar theoretical lenses to examine presence in the distinct VR context.
Another theoretical contribution is the testing of the research model explaining users' engagement in the distinctive context of VR. To the best of our knowledge, this study is among the first to theorize about features of presence and user engagement in the business application of VR. Although the relationships in our research model have been examined in the online commerce context before, this is the first empirical study that we are aware of to test these relationships in the highly immersive VR setting. This research offers additional insights into how two major features of VR impact the underlying mechanisms in engaging VR users, particularly, the path from spatial presence to engagement is in the opposite direction as predicted.

\subsection{Practical contributions}

Our findings provide practitioners with a set of interesting insights in how to improve users' engagement in VR applications. Our study advises practitioners to incorporate features related to presence, especially spatial presence, since it can better deliver hedonic value to VR users. For example, by simulating the physical environment and interactions of actual stores more precisely, practitioners can enhance users' emotional states in VR. In addition, the results suggest that an increasing level of spatial presence or reality of the physical world might not necessarily result in engagement. Therefore, practitioners would need to find an optimal level of reality in the virtual environment that generates the highest level of engagement. For example, adding a layer of digital information into a virtual environment may decrease reality but would be more engaging as found in augmented reality literature [39].

Furthermore, our study suggests that to better engage users, practitioners should focus on delivering utilitarian value to VR users. In fact, in the business context, developers may increase the utilitarian value of VR applications by designing features to enrich product features and information. This is because engagement appears to be more affected by utilitarian value than hedonic value. In fact, business users of VR may interact with the applications with the primary goal of usefulness.

\subsection{Limitations, future research directions, and conclusion}

As with any research, the findings of our study should be interpreted with certain limitations. First, our experiment is based on a small sample size, which 
may result in the nonsignificant relationships in the research model. Additional measurement is recommended to replicate this study with a larger sample size. Second, our results suggest a negative relationship between spatial presence and engagement. Therefore, it is necessary to investigate this relationship in future research to find evidence for the formation of this relationship. Third, our study aims to explore VR in the business context and may have been affected as such. Therefore, future research is encouraged to investigate presence in a different context. Fourth, while our study uses a robot assistant to manipulate the degrees of social presence, the robot did not directly interact with the subjects when they performed the experiment task. Consequently, a more sophisticated stimulus of social presence is necessary to be developed to better understand how different levels of social presence impact users' perceptions.

While there is little prior knowledge explaining the effects of presence on user responses in the VR context, we explored possible explanations of the underlying mechanisms of how a VR application engages users in the business context. Our study provides empirical evidence that the two dimensions of presence can be effective predictors of engagement and other behavioral responses. These findings serve as a starting point for researchers to gain a broader understanding of the factors influencing user responses in using business VR applications.

\section{References}

[1] H. Williams, "What industries are using virtual reality?", ComputerWorld UK, March 23, 2017, retrieved from https://www.computerworlduk.com/applications/sixbusiness-uses-for-virtual-reality-3641742/

[2] M.V. Sanchez-Vives and M. Slater, "From Presence to Consciousness through Virtual Reality", Nature Reviews Neuroscience, 6, 2005, p. 332. doi:10.1038/nrn1651

[3] S. Bose, " $\$ 17.8$ Billion in Business Investment Expected in AR and VR This Year, Is Your Business Ready?", 2018, retrieved from https://smallbiztrends.com/2018/04/howbusinesses-can-use-augmented-reality.html

[4] N. Padmanaban, R. Konrad, T. Stramer, E.A. Cooper, and G. Wetzstein, "Optimizing Virtual Reality for All Users through Gaze-contingent and Adaptive Focus Displays", Proceedings of the National Academy of Sciences, 2017. doi.org/10.1073/pnas.1617251114

[5] S.W. Greenwald, A. Kulik, A. Kunert, S. Beck, B. Fröhlich, S. Cobb, . . . and P. Maes, "Technology and Applications for Collaborative Learning in Virtual Reality", paper presented at the Making a Difference: Prioritizing Equity and Access in CSCL, 12th International Conference on Computer Supported Collaborative Learning (CSCL), 2017, Philadelphia, PA.

[6] M. Marquess, S.P. Johnston, N.L. Williams, C. Giordano, B.E. Leiby, M.D. Hurwitz, . . , and R.B. Den, "A Pilot Study to Determine if the Use of a Virtual Reality Education Module Reduces Anxiety and Increases Comprehension in Patients Receiving Radiation Therapy", Journal of Radiation Oncology, 6(3), 2017, pp. 317-322. doi:10.1007/s13566-017-0298-3

[7] N. Staggers and A.F. Norcio, "Mental Models: Concepts for Human-computer Interaction Research", International Journal of Man-Machine Studies, 38(4), 1993, pp. 587-605. doi:https://doi.org/10.1006/imms.1993.1028

[8] K.M. Lee, "Presence, Explicated", Communication Theory, 14(1), 2004, pp. 27-50. doi:doi:10.1111/j.14682885.2004.tb00302.x

[9] M.L. Ryan, Narrative as Virtual Reality: Immersion and Interactivity in Literature and Electronic Media, Johns Hopkins University Press, 2001.

[10] F. Biocca, C. Harms, and J.K. Burgoon, "Toward a More Robust Theory and Measure of Social Presence: Review and Suggested Criteria", Presence: Teleoperators and Virtual Environments, 12(5), 2003, pp. 456-480. doi:10.1162/ 105474603322761270

[11] H. Van Kerrebroeck, M. Brengman, and K. Willems, "When Brands Come to Life: Experimental Research on the Vividness Effect of Virtual Reality in Transformational Marketing Communications", Virtual Reality, 21(4), 2017, 177-191. doi:10.1007/s10055-017-0306-3

[12] J.M. Loomis, "Distal Attribution and Presence", Presence: Teleoperators and Virtual Environments, 1(1), 1992, pp. 113-119. doi:10.1162/pres.1992.1.1.113

[13] W.A. Ijsselsteijn, H. de Ridder, J. Freeman, and S.E. Avons, "Presence: Concept, Determinants, and Measurement", Proceedings of the SPIE-The International Society for Optical Engineering, November 2000. doi: $10.1117 / 12.387188$

[14] J.V. Draper, D.B. Kaber, and J.M. Usher, "Telepresence", Human Factors, 40(3), 1998, pp. 354-375. doi:10.1518/001872098779591386

[15] J. Fulk, C.W. Steinfield, J. Schmitz, and J.G. Power, "A Social Information Processing Model of Media Use in Organizations", Communication Research, 14(5), 1987, pp. 529-552. doi:10.1177/009365087014005005

[16] K.S. Hassanein and H.M. Milena, "Manipulating Perceived Social Presence Through the Web Interface and Its Impact on Attitude Towards Online Shopping", 
International Journal of Human-Computer Studies, 65(8), 2007, pp. 689-708.

[17] D. Cyr, K. Hassanein, M. Head, and A. Ivanov, "The Role of Social Presence in Establishing Loyalty in e-Service Environments", Interacting with Computers, 19(1), 2007, pp. 43-56. doi:10.1016/j.intcom.2006.07.010

[18] E. Deci and R.M. Ryan, "Intrinsic Motivation and SelfDetermination in Human Behavior, Plenum Press, New York, NY, USA, 1985.

[19] J. Gutman, "Means-end Chains as Goal Hierarchies", Psychology \& Marketing, 14(6), 1997, pp. 545-560. doi:10.1002/(SICI)1520-6793(199709)14:6<545::AID-

MAR2>3.0.CO;2-7

[20] H. Woodruffe-Burton, S. Eccles, and R. Elliott, "Towards a Theory of Shopping: A Holistic Framework", Journal of Consumer Behaviour, 1(3), 2002, pp. 256-266. doi:doi:10.1002/cb.71

[21] S.A. Eroglu, K.A. Machleit, and L.M. Davis, "Empirical Testing of a Model of Online Store Atmospherics and Shopper Responses", Psychology \& Marketing, 20(2), 2003, pp. 139-150. doi:doi:10.1002/mar.10064

[22] D.R. Fortin and R.R. Dholakia, "Interactivity and Vividness Effects on Social Presence and Involvement with a Web-based Advertisement", Journal of Business Research, 58(3), 2005, pp. 387-396.

[23] J.B. Kim, "The Mediating Role of Presence on Consumer Intention to Participate in a Social Commerce Site", Journal of Internet Commerce, 14(4), 2015, pp. 425454. doi:10.1080/15332861.2015.1092067

[24] C.M. Chiu, E.T.G. Wang, Y.H. Fang, and H.Y. Huang, "Understanding Customers' Repeat Purchase Intentions in B2C E-commerce: the Roles of Utilitarian Value, Hedonic Value and Perceived Risk", Information Systems Journal, 24(1), 2014, pp. 85-114. doi:doi:10.1111/j.13652575.2012.00407.x

[25] S. Kim and M.S. Eastin, "Hedonic Tendencies and the Online Consumer: An Investigation of the Online Shopping Process", Journal of Internet Commerce, 10(1), 2011, pp. 68-90. doi:10.1080/15332861.2011.558458

[26] A. Mollen and H. Wilson, "Engagement, Telepresence and Interactivity in Online Consumer Experience: Reconciling Scholastic and Managerial Perspectives", Journal of Business Research, 63(9), 2010, pp. 919-925. doi.org/10.1016/ j.jbusres.2009.05.014

[27] A.M. Fiore, J. Kim, and H.H. Lee, "Effect of Image Interactivity Technology on Consumer Responses Toward the Online Retailer", Journal of Interactive Marketing, 19(3), 2005, pp. 38-53. doi:doi:10.1002/dir.20042
[28] I.P. Tussyadiah, D. Wang, T.H. Jung, and M.C. tom Dieck, "Virtual Reality, Presence, and Attitude Change: Empirical Evidence from Tourism", Tourism Management, 66, 2018, pp. 140-154. doi:https://doi.org/10.1016/ j.tourman.2017.12.003

[29] D. Shin, "Empathy and Embodied Experience in Virtual Environment: To What Extent Can Virtual Reality Stimulate Empathy and Embodied Experience?", Computers in Human Behavior, 78, 2018, pp. 64-73. doi:https://doi.org/10.1016/ j.chb.2017.09.012

[30] M. Csikszentmihalyi, Beyond Boredom and Anxiety, Jossey-Bass, San Francisco, CA, 1975.

[31] R. Algharabat, N.P. Rana, Y.K. Dwivedi, A.A. Alalwan, and Z. Qasem, "The Effect of Telepresence, Social Presence and Involvement on Consumer Brand Engagement: An Empirical Study of Non-profit Organizations", Journal of Retailing and Consumer Services, 40, January 2018, pp. 139-149. doi:https://doi.org/10.1016/j.jretconser.2017.09.011

[32] R.M. Held and N.I. Durlach, "Presence: Teleoperators and Virtual Environments", Telepresence, 1(1), 1992, pp. 109-112. doi:10.1162/pres.1992.1.1.109

[33] T.L. Childers, C.L. Carr, J. Peck, and S. Carson, "Hedonic and Utilitarian Motivations for Online Retail Shopping Behavior. Journal of Retailing, 77(4), 2001, pp. 511-535. doi: 10.1016/s0022-4359(01)00056-2

[34] H.L. O'Brien, "The Influence of Hedonic and Utilitarian Motivations on User Engagement: The Case of Online Shopping Experiences", Interacting with Computers, 22(5), 2010, pp. 344-352. doi:10.1016/j.intcom.2010.04.001

[35] R.R. McCrae and P.T. Costa, "Joint Factors in Selfreports and Ratings: Neuroticism, Extraversion and Openness to Experience", Personality and Individual Differences, 4(3), 1983, pp. 245-255. doi:https://doi.org/10.1016/0191-8869(83)90146-0

[36] S.J. Ahn, et al., "Experiencing Nature: Embodying Animals in Immersive Virtual Environments Increases Inclusion of Nature in Self and Involvement With Nature", Journal of Computer-Mediated Communication, 21(6), 2016. 21(6), pp. 399-419.

[37] Z. Zhou, et al., "Attracted to or Locked In? Predicting Continuance Intention in Social Virtual World Services", Journal of Management Information Systems, 29(1), 2012, pp. 273-306.

[38] E.N. Wiebe, et al., "Measuring Engagement in Video Game-Based Environments: Investigation of the User Engagement Scale", Computers in Human Behavior, 32, 2014, pp. 123-132.

[39] C. Dede, "Immersive Interfaces for Engagement and Learning", Science 323(5910), 2009, pp. 66-69. 
[40] J.F. Hair, R.L. Tatham, R.E. Anderson, and W.C. Black, Multivariate Data Analysis, Prentice Hall, Englewood Cliffs, NJ, 1995.

[41] G. Bente, S. Rüggenberg, N.C. Krämer, and F. Eschenburg, "Avatar-Mediated Networking: Increasing Social Presence and Interpersonal Trust in Net-Based Collaborations", Human Communication Research, 34(2), 2008, pp. 287-318. doi:10.1111/j.1468-2958.2008.00322.x

[42] J. Hamari, D.J. Shernoff, E. Rowe, B. Coller, J. AsbellClarke, and T. Edwards, "Challenging Games Help Students Learn: An Empirical Study on Engagement, Flow and Immersion in Game-based Learning", Computers in Human Behavior, 54, 2016, pp. 170-179. doi:https://doi.org/10.1016/ j.chb.2015.07.045

[43] E. Baldi and C. Bucherelli, "The Inverted "U-Shaped" Dose-Effect Relationships in Learning and Memory: Modulation of Arousal and Consolidation. Nonlinearity in Biology", Nonlinearity in Biology, Toxicology, Medicine, 3(1), 2005, pp. 9-21. doi:10.2201/nonlin.003.01.002

[44] F.D. Davis, "Perceived Usefulness, Perceived Ease of Use, and User Acceptance of Information Technology", MIS Quarterly, 13(3), 1989, pp. 319-340. 